\title{
DESIGN AND IMPLEMENTATION OF A FIXED SYSTEM FOR VIDEO-ACOUSTIC MONITORING OF URBAN NOISE POLLUTION
}

\author{
NEDEFF, V[alentin]; MOSNEGUTU, E[milian]; TOMOZEI, C[laudia]; \\ PAL, A[nton] \& SAMOILA, F[lorin]
}

\begin{abstract}
The intensification of transport in the urban environment has generated an increase oflevel noise. Therefore, the EC has implemented an environmental policy for the reducing the noiselevel. Thus, the limit values acceptable for the noise levels in urban areas are $50 \mathrm{~dB}$. To determine the noise level for these environments were designed and made various systems to measure level of noise, the majority of them being portable.

S.C. Aerostar S.A. in collaboration with the "VasileAlecsandri" University of Bacau has designed and developed a system for acoustic-video monitoring. It consists of two components: system fixed of video-acoustic monitoring located in different areas of the city; the dispatcher that retrieves and processes obtained data for making noise maps and generate alarm reports. In addition to other noise monitoring components systems, the proposed system also has a surveillance room with auto positioning software that aims to identify the source of noise pollution.
\end{abstract}

Keywords:acoustic and video monitoring, urban noise

\section{INTRODUCTION}

Urban population is exposed to a complex mixture of environmental pollutants [9]. The noise pollution has emerged relatively recently, due to technological development as one of the aggressive factors acting on the hearing (and not only) and invaded the life and the peace of people.

Intensification transport of all types (road, rail and air), increasing the number of industrial units and housing with a growing number of appliances have a negative impact on livelihoods, by producing noise pollution.

In the urban area in most areas, the noise was always present, but often under estimated $[4,6,7]$.

Of all types of sources noise, the traffic noise is a major source of pollution environmental on the around the globe, both in the developed countries and under developed (Fig.1) [2, 5, 8].

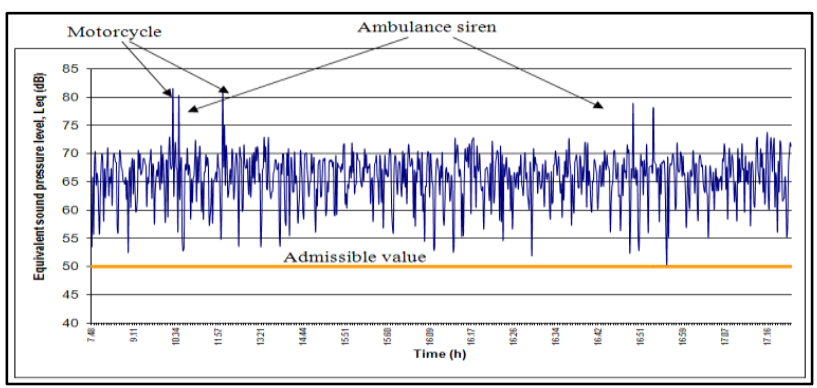

Fig. 1. The values variation of the equivalent sound pressure level, $\mathrm{L}_{\text {Aeq, }}$, recorded in a crowded urban area [3]
The noise pollution in cities becomes a growing problem because the urban environment is becoming increasingly crowded and busy [4, 5, 6].

Given these studies the EU has gradually developed its policy regarding noise. In the second environmental program (1977-1981) it was intended to introduce a noise reduction plan based on taxes and aimed at improving environmental quality. This ambitious plan was not implemented until 1986 (European Single Act) because the European Community was less involved in environmental policy and hence in the noise policy.

In Europe, people are exposed frequently to noise levels above $65 \mathrm{~dB}(\mathrm{~A})$, leading to adversely affect human health. The population complaints about noise have increased since 1992. Around 100 million Europeans suffer because the noise levels that are considered unacceptable. Also, in the United States, seven of every 100 people reported hearing problems. Globally, about 120 million people were evaluated and diagnosed with hearing impairments $[4,7,10]$.

In Romania, in 2005 through HG no. 321 published in M.O. no. 358/2005, amended and completed by Government Resolution no. 674 of June 28, 2007, were introduced legislative regulations on the evaluation and management of environmental noise. These approaches at national, require avoiding, prevent or reduce the harmful effects caused by ambient noise, including annoyance, by implementing the following measures:

a.Determination of exposure to environmental noise through the noise mapping;

b.Providing information to the public on environmental noise and its effects;

c.Adoption, based on the noise maps, of action plans, aimed at preventing and reducing environmental noise where necessary, particularly levels of exposure that can have harmful effects on human health.

In accordance with the standards that define levels of noise are presented the basic technologies for measuring and defining different types of noise sources. The most common sources of noise are:

- The traffic noise (road, rail and air);

- Industrial noise (from factories, power stations etc.)

- Recreational noise (stadiums, parks etc.)

- Domestic noise;

- Noise from construction sites.

- The equipment used to measure noise may be:

- Different types of sound level meters;

- Portable monitoring stations; 
- Devices for measuring and recording noise of type the noise monitoring terminals.

After the measurement of the noise level is monitoring its dispersion in the environment, taking into account the artificial or natural obstacles, to achieve an acoustic mapping.

The zonal acoustic mapping allows, among other things, the quantification the level of noise in the area studied by $[1,11]$ :

- Evaluation of population exposure;

- Creating a database for urban planning with zoning activities noisy and the sensitive areas;

- Modelling of different scenarios for future evolution of the noise levels;

- Impact predictions of the noise on infrastructure designed and in industrial activities.

Elaboration of noise maps can be made using noise values obtained by real measurement in the points previously established using predictive models or simulations. Another modality consists in using a mixed system of simulations which are completed and verified by values obtained from real measurements $[1,11]$.

The acoustic mapping allows creating noise mapping starting with input data (GIS or AutoCAD files for construction and topography, data about traffic, aerial scanning to find the height of buildings), entering values obtained by measurements at predetermined locations and ending with the generation of noise map.

Besides assessing the current situation can analyse various virtual situations, possible in the future.

The main stages of achieving of noise maps are:

1.Achieve the GIS map, pictures or cadastral map;

2.The modelling in the specialized computer program on the data of urban zone (this is done on the base of noise mapping where is required the knowledge of the building height);

3.Information about road traffic, railway, airport, industrial area - number vehicles/hour, categories of vehicles, averages speed, distribution at 24 hours on the 3 time intervals;

4.Calculation of noise distribution in urban area, using the mentioned program. Until now map can be achieved only by computer simulation. The result of simulation obtained after the processing data, is just theoretical and can be validated by specific acoustic measurements.

5. The validation of result is achieved by determining the noise level in different places and then followed by automatic or manual updating of the map, to get concordance between the simulation and the real data. A more accurate result requires a large number of determinations.

Vast majority of urban noise monitoring systems are aimed to the noise measurement but identification of the noise source is imprecise. For an exact identification of the noise source, to the actual acoustic monitoring systems must be brought new components aimed at:

-The video identification of the noise source;
-The software to rotate the video camera to the noise sourcenoise;

-The software to determine the distance to the pollution source;

-Creation of fixed terminals for monitoring and dispatcher.

For the monitoring acoustic-video of the urban noise, SC Aerostar S.A. in collaboration with "VasileAlecsandri" University of Bacau have designed and developed anacoustic-video monitoring fixed system.

The acoustic-video monitoring systems, mobile or fixed, are usually specific for purpose which they were designed. The difficulties in designing such a system were related to relatively few information about such systems, at which can add of their specificity given the fact that were individually designed as a result of the monitored activity.The video-acoustic monitoring aims to identify environmental problems caused by noise, the generation of the noise mapsidentifying problem areas and the noise sources that exceeded the admissible limit values and adoption of appropriate measures to reduce urban noise.

The paper presents a way to achieve such a system for the video-acoustic monitoring adapted to zone monitoring requirements. The designed system can be support in the future related activities and in the same time can be basis for the future development in the proposed area and for other areas. It provides information regarding of the noise level then processes the dates in the noise maps and it generates alarms.

\section{THE DESIGNING OF THE ACOUSTIC - VIDEO MONITORING SYSTEM}

The components of the acoustic-video monitoring systemare as follows [12].

\subsection{Equipment of monitoring video-acoustic for noise pollution -EMVANP (Fig. 2)}

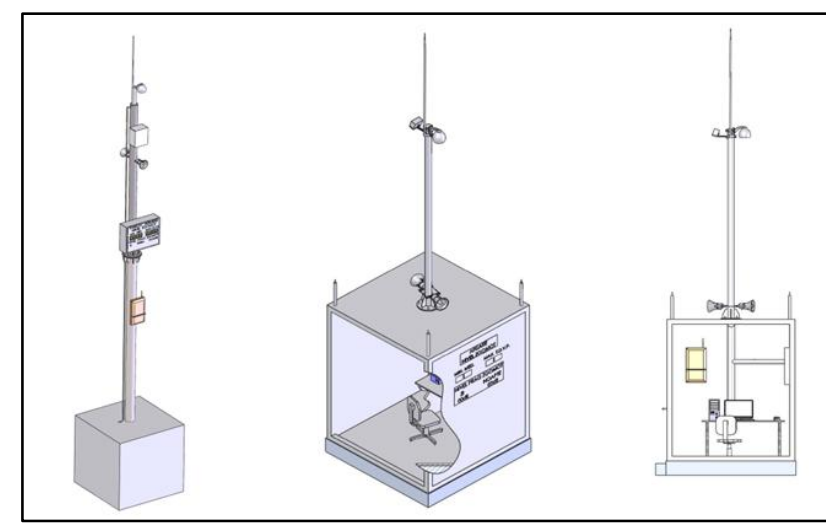

Fig. 2. EMVANP - Equipment of monitoring video-acoustic for noise pollution [12]

The fixed station of monitoring video-acoustic, prototype, contains the following functional components: A. Video block with transmission system through the optic fiber (O.F.) which contains: video camera of type speed dome (Fig. 3 and Fig. 4), which captures images of the monitored environment; the transmitter O.F., of type 
A-172, which transmitting the image and the commands to the video camera and the video images to the dispatcher (Dispatcher for the monitoring video-acoustic of the noise pollution - DMVANP); transmitter-receiver of optical fiber (transceiver for O.F.), through which it convey the data signals (display and warning) between the computer block and dispatcher (DMVANP); power sources; the protection modules, type of protection system for conveyance (P.S.C.), video system protection (V.S.P.) and protection system for power source (P.S.P.S.) at video camera; detector motion for surveillance and alarming in case of unauthorized access to the system EMVANP, of type SDI-77XL-DIR-LT; rod captured of the electrostatic protection of EMVANP, cables connection; metal box of protection of the majority components listed above.

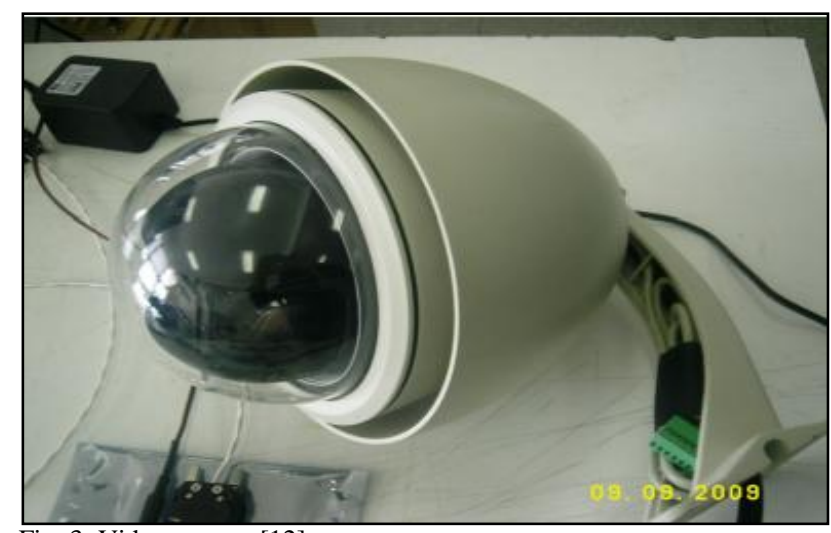

Fig. 3. Video camera [12]

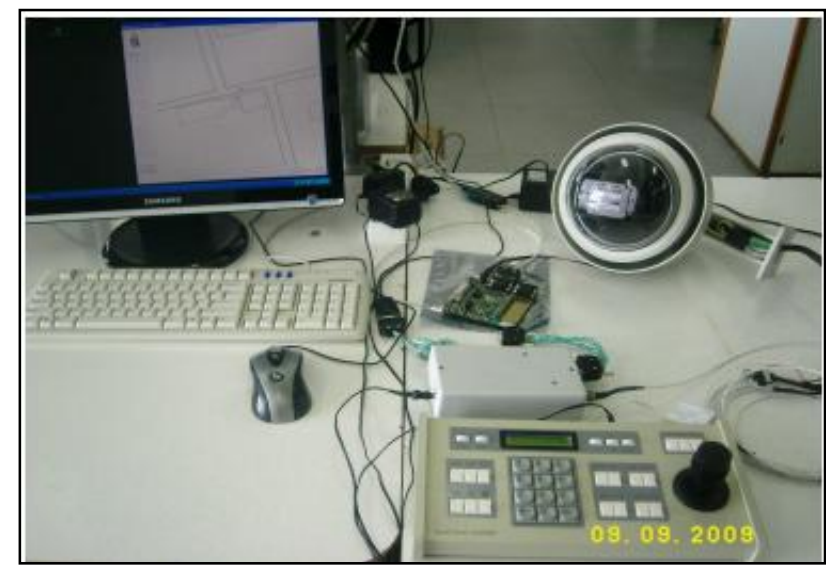

Fig. 4. The montage for control system testing of the video camera [12]

B. Computer block, with display and warning who includes: computer block of the position (Fig. 5); power transformer; junction block microphones; network of microphones: MICR 1, MICR 2, MICR 3, MICR 4 (Fig. 6 ); power source of low frequency amplifier (PSLFA); low frequency amplifier (LFA); network of loudspeakers: SPEAKER 1, SPEAKER 2; plaque junction (PJ); plaque junction display (PJD); display of noise parameters (DNP) (Fig. 7); power source (PS); plaque junction of network (PJN) c.a.; automatic switch from network; cables connection; protective box.

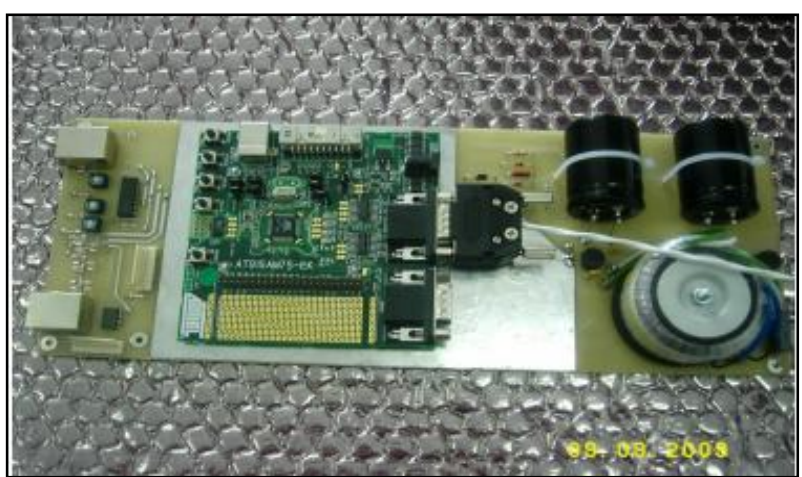

Fig. 5. Calculator of the noise source position [12]

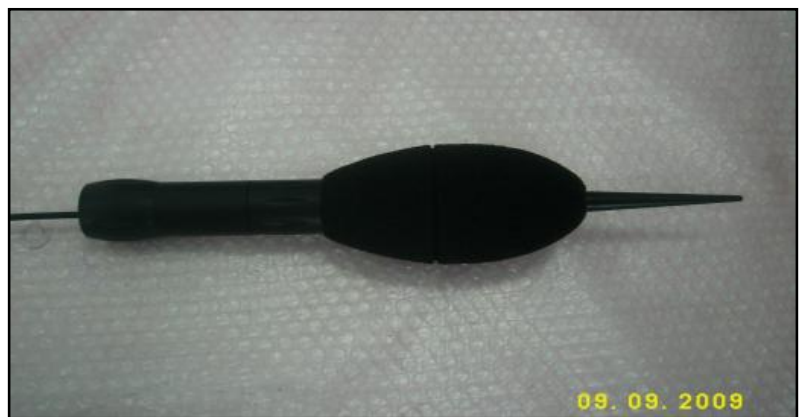

Fig. 6. Microphone, type 4952[12]

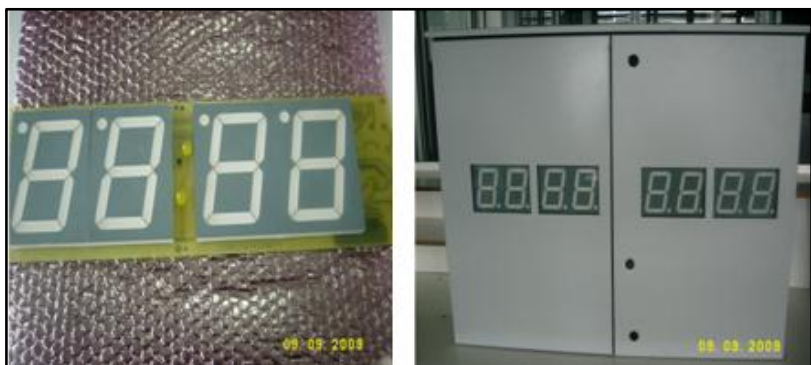

Fig. 7. Display for the noise parameters [12]

C. Noise Monitoring Terminal, type 3639-E200. The noise monitoring terminal captures through its microphone the ambient noise (Fig. 8), calculated and stored in internal memory the noise parameters, which we send to server, located in dispatcher.

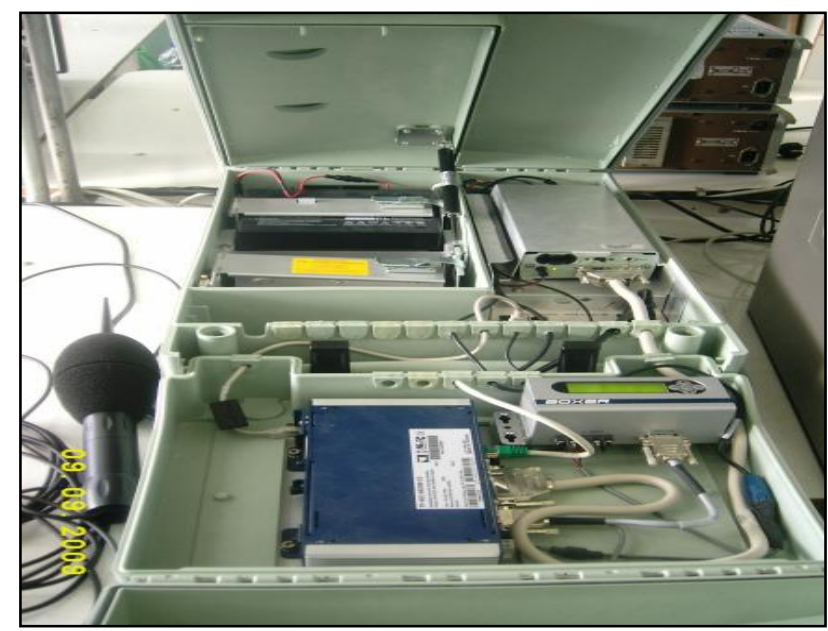

a) 


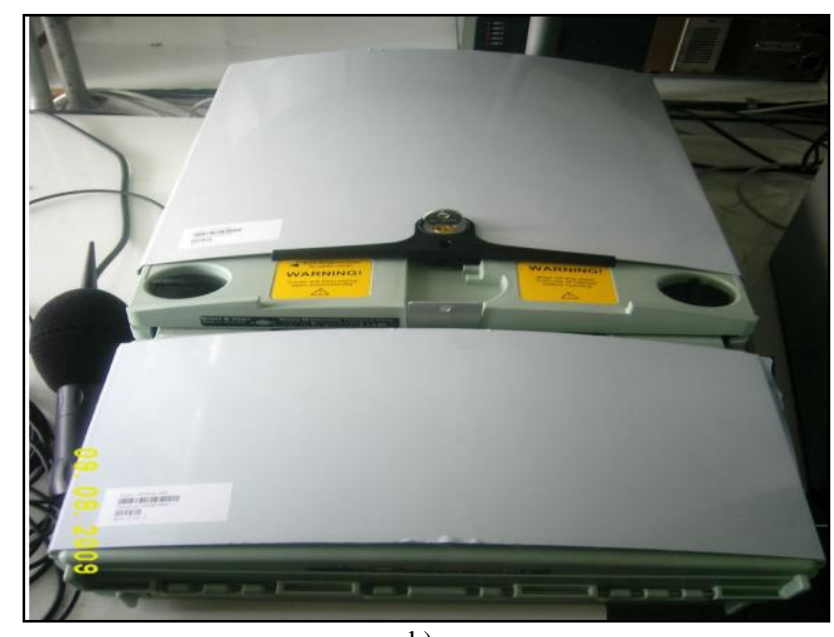

b)

Fig. 8. Environmental Monitoring Terminal [12]:a) Interior view; b) Exterior view

\section{Tripod (support)}

The container is fitted all functional blocks:

- Video camera;

- Detector of motion;

- Rod captured.

In the outside, on the support, are attached the following items:

- Networkof microphones: MICR 1, MICR 2, MICR 3, MICR 4;

- Networkof speakers: SPEAKER 1, SPEAKER 2;

- Cablesfor connection.

The container provides a protection of the equipment's towards environmental factors and working conditions for staff involved in maintenance activities and for the operation activities.

\subsection{Dispatcher for the monitoring video-acoustic of the noise pollution - DMVANP (Fig. 9)}

The dispatcher is the system component that centralizes all data captured by the work equipment, information on noise, images, events and alarms.

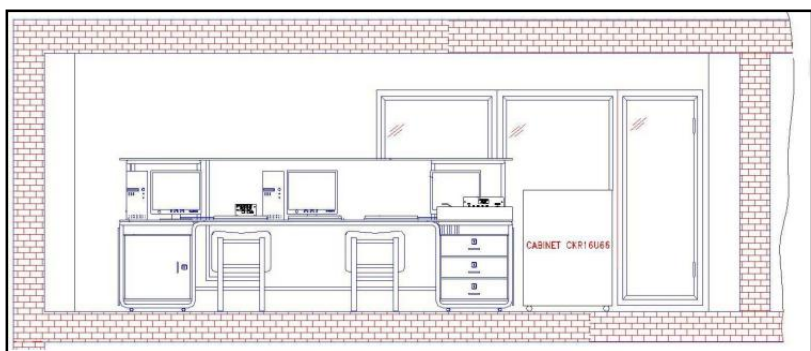

Fig. 9. Dispatcher (DMVANP) - possible configuration [12]

In the prototype stage, DMVANP contains the following components functional:

A. AUDIO MODULE, which can generate the warning vocal message, for the each equipment, in situations of intentional noise pollution, unauthorized access, antisocial acts etc. The microphone of audio module perform the duties of voice operator transducer into electrical signals which are processed (amplified, compressed) and transmitted for the equipment, selectively, depending on the needs of warning.
B. BLOCK OF SIGNALS JUNCTION, whose function is of interface at connector's level.

C. ADAPTER RS 232/RS485, to convert the signals of data from RS232 to RS485.

D. SERVER OF NOISE MONITORING, (Fig. 10) performs several functions, including:

- Database for the noise recordings using the specific equipment;

- Database for the calculated noise parameters;

- Real time monitoring of the noise evolution and graph displaying with the levels of noise;

- Medium to generate of the noise reports;

- Medium to achieve the noise maps;

- Medium to noise prediction;

- Medium to achievement measures plans and action in managing of noise pollution;

- The equipment management for noise monitoring.

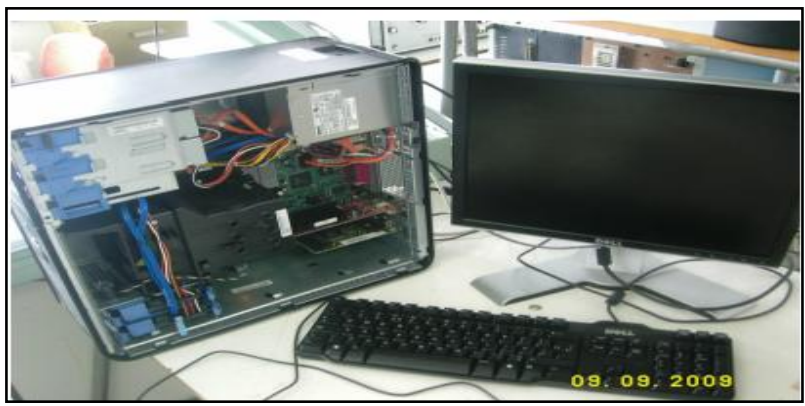

Fig. 10. Server of noise management [12]

E. PC POSITION DISPLAY is a computer for the information management on the position of noise sources (Fig. 11). Position of the noise sources is determined by computer using noise monitoring equipment. The display shows the map (the plan) (Fig. 12) with the positions of noise sources in compared with the location of the monitoring equipment and the dominant levels measured.

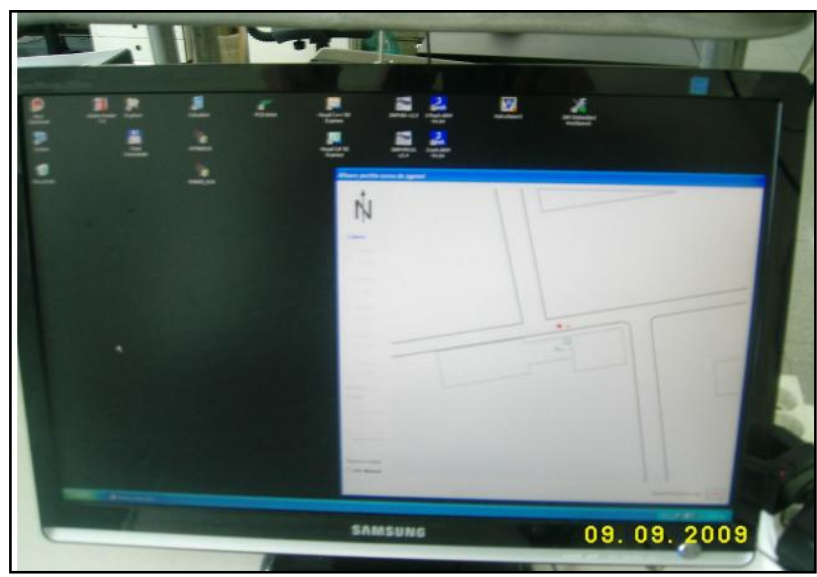

Fig. 11. The display for the noise source position [12] 


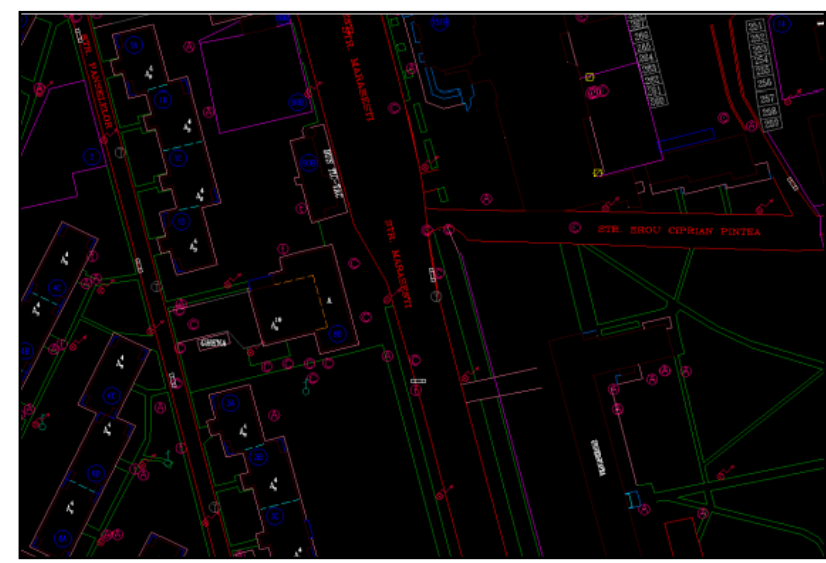

Fig. 12. Cadastral plan of themonitored area [12]

F. REGISTERED DIGITAL VIDEO (Fig. 13) (DVR), type SCB5004N264, performs the following functions:

- Storing of the video signals provided by video cameras;

- Recording of the noise signals associated on each video camera synchronous with the image signals;

- Processing the data from the database with information of image and associated sound;

- Recording of alarm data generated by motion detectors, and warning of operator by generating an alarm signals (sound, video) in real time;

- Generation of reports through the alarms.

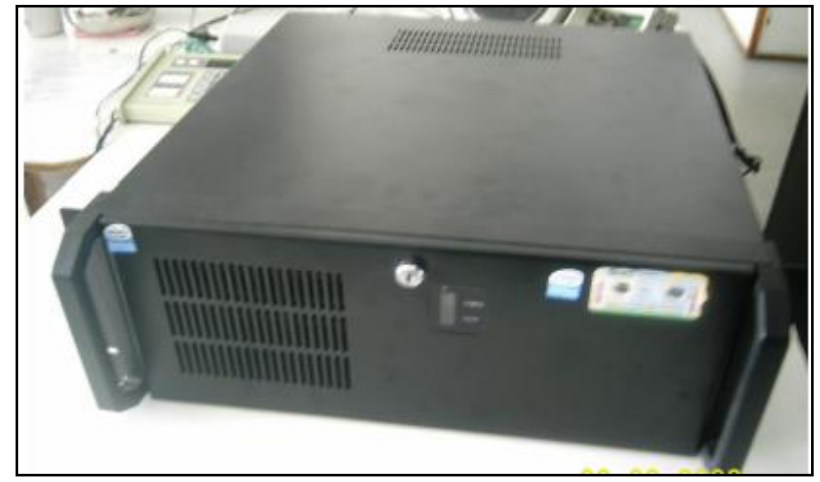

Fig. 13.Video digital recorder [12]

G. CONSOLE, type KS 105, to control the video cameras (Fig. 14).The server interfaces with the operator are achieved through a display (Monitor Noise Maps), a keyboard (Keyboard 2) and a mouse (Mouse 2).

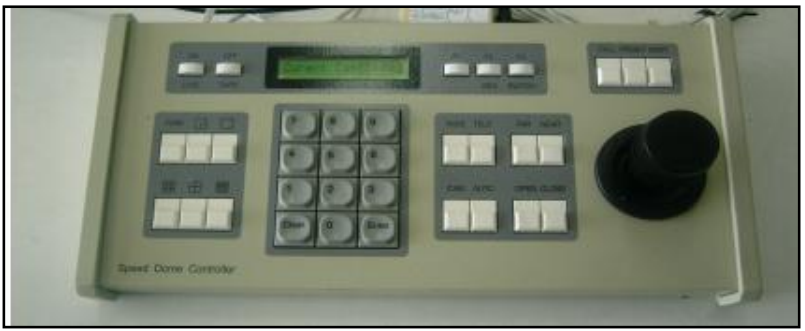

Fig. 14. The console of video camera command [12]

H. TRANSCEIVER O.F.,type A721CM provides the data communication with the monitoring equipment, on the optic fiber, for the following electrical signals:

- Contactsmotion detector;

- Thesignal of audio-frequency (PSLFA), vocal warning;
- Thesignal of noise captured in the supervised environment (LFA MICR 4) and is associated with video signals;

- Thesignal of data from the noise monitoring terminal;

- Thesignal of data with the noise source position;

- Thesignal of data for display of the noise parameters.

I. RECEIVER O.F., type A174, provides communication of data and the video signal from the camcorder at the DVR.

\section{J. POWER SOURCE.}

K. WELDING BOX O.F., provides the routes junction of the optic fiber at the cable connection with the noise monitoring equipment.

L. UNINTERRUPTED POWER SOURCE (UPS), through which is supplied the server 3642 and the digital video recorder.

M. OFFICE, in which installs the following blocks:

-Digitalvideo recorder;

-TransceiverO.F., type A $721 \mathrm{CM}$

-ReceiverOF, type A174;

-Powersource, type P 405;

-Themodule of data conversion, type SCB 16004;

-UPS, type SUA1500RMI2U.

The other components blocks of the dispatcher were mounted on the tables.

\subsection{Lines of the data transmission and for the power supply}

For the data transmission and for electrical networks used for the acoustic-video monitoring system was chosen as the optimal technical solution the same routes of optic fiber cables. These cables can be mounted air or buried, provided with protective tubes to destructive action environmental, and with steel cables.

\section{SOFTWARE TO COMMANDTHE VIDEO CAMERA}

To identify video of the noise source was made a software that to order the rotation of video camera in the direction from which the noise is emitted.

The program reads a command (or several) from a serial port and sends command guidance through the serial port to one or more cameras. Then displays the status of receipt of the orientation command from port. If it is valid it shall be returned on the properly format at the camera connected to serial. The video camera is focused on the azimuth in the range $0 \ldots 360$ degrees. This interval is divided into 16 equal zones.

It also allows selecting the output and input COM to camera via a configuration menu. Will disable in the same time certain indicators. The program does not check the COM, this task is for user and/or system administrator

The current version allows up to 6 indicators. Program structure allows an unspecified number of indicators. 
To control the communication on the video camera was chosen Pelco.D protocol. The camera has 16 preset positions.

\subsection{The logic structure of software}

The program is divided into several classes, predefined (type: form, checkboxlist, combobox, button) but some classes are constructed.

It was constructed two classes: indicator (encapsulates all options for association COM and graphics display method) and indicator_conf (facilitates the transfer of information between configuration window of COM associations and the panel of indicators).

The classes for transmission and message formatting to COMs are SerialPort and Pelco.D. SerialPort is a predefined class that can read or write on the serial port. This class allows: full setup of serial port, the opening operations, the closing, reading, writing of the port in various formats. In the application COMs are opened only if the associated indicator is active.

The class Pelco.D permitted only formatting of message to the video camera but not the transmission on serial.

It was specified a corresponding time of timedout for reading and writing so that the program does not freeze in the readout on ports. Additionally it was specified a reasonable period of waiting and adjustable for a good communication in time.

\subsection{The interface of the software}

The program uses an intuitive interface, in addition on the classic Windows software, there is a interface of the display indicator (Fig. 15).

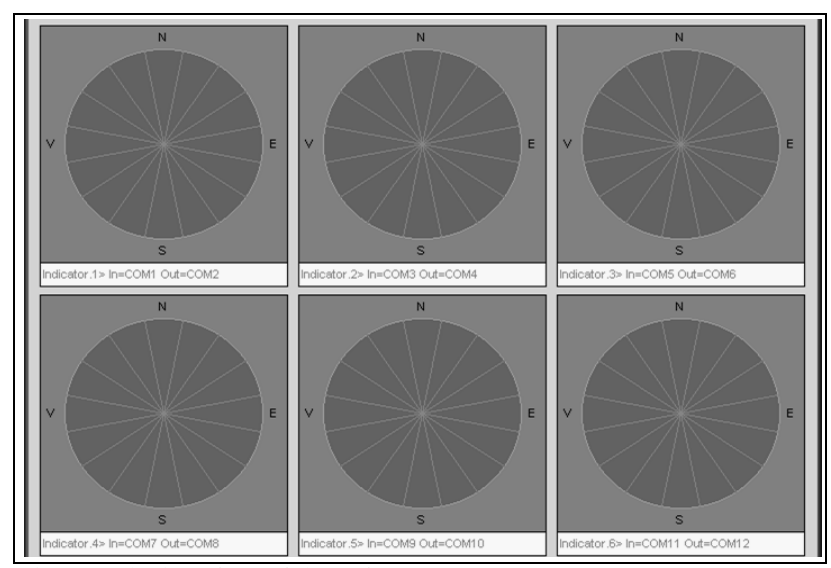

Fig. 15. The interface of the software

The indicator is composed of an area to indicate the direction of the video camera filming. The area is a sector of a circle with yellow light. It is assumed that the north is associated with the 0 , the east with 3 , the south with 7 and the west with 11 . The number for the yellow area is shown on this area.

\subsection{The code of the software}

The program was written in Visual $\mathrm{C \#}$ and compiled with Visual Studio 2005.

\section{CONCLUSION}

Following the analysis of the work described above can be drawn the following conclusions:

- The noise pollution is one of the aggressive pollution factors with the significant impact on environmental;

- Of all types of noise, traffic noise is a major source of pollution;

- Dueto the results obtained of analysis performed on the noise pollution, the EU developed an environmental policy related to reducing noise;

- Todetermine the urban noise different types of devices are used and with the values obtained can made noise maps;

- S.C. Aerostar S.A. in collaboration with "VasileAlecsandri" University of Bacau have designed and implemented a fixed noise monitoring, consisting of fixed monitoring stations and a dispatcher;

- Measurement of noise with fixed stations allows continuous monitoring of noise levels to verify compliance with regulations;

- Thefixed monitoring stations, compared to classical systems, propose a video identification system of noise sources.

\section{REFERENCES}

[1] Stephan Weber, Tom Litschke, (2008), Variation of particle concentrations and environmental noise on the urban neighbourhood scale, Atmospheric Environment,No. 42, pp. 7179-7183

[2] Olayinka S. Oyedepo, Abdullahi A. Saadu, (2010), Evaluation and analysis of noise levels in Ilorin metropolis, Nigeria, Environmental Monitoring and Assessment, No. 160, pp. 563-577

[3] Paulo Henrique Trombetta Zannin, (2006), Occupation al noise in urban buses, International Journal of Industrial Ergonomics, No. 36, pp. 901-905

[4] Samira Ibrahim Korfali, May Massoud, (2003), Assessment of Community Noise Problem in Greater Beirut Area, Lebanon, Environmental Monitoring and Assessment, No. 84, pp. 203-218

[5] Hakan Doygun, Derya Kuşat Gurun, (2008), Analysing and mapping spatial and temporal dynamics of urban traffic noise pollution: a case study in Kahramanmaraş, Turkey, Environmental Monitoring and Assessment,No. 142, pp. 65-72

[6] Paulo Henrique Trombetta Zannin, Andressa Maria Coelho Ferreira and Bani Szeremetta, (2006), Evaluation of Noise Pollution in Urban Parks, Environmental Monitoring and Assessment,No. 118, pp. 423-433

[7] Samuel Barsanelli Costa, Roberto Wagner Lourenço, (2011), Geo-processing applied to the assessment of environmental noise: a case study in the city of Sorocaba, São Paulo, Brazil, Environmental Monitoring and Assessment, No. 172, pp. 329-337

[8] Nedeff Valentin, Tomozei Claudia, Panainte Mirela, Macarescu Bogdan, Mosnegutu Emilian, Lazar Gabriel,(2011), Noise monitoring in a high traffic area of Bacau, Romania, Journal of Engineering Studies and Research, Alma Mater Publishing House, Bacau,Vol. 17, No. 1, pp. 57-63

[9] Tang U.W., Wang Z.S., Influences of urban forms on trafficinduced noise and air pollution: Results from a modelling system, Environmental Modelling \& Software (2007), No. 22, pp. 17501764

[10] Fernando Augusto de Noronha Castro Pinto, Maysa Daniela Moreno Mardones, (2009), Noise mapping of densely populate neighborhoods - example of Copacabana, Rio de Janeiro - Brazil, Environmental Monitoring and Assessment,No.155, pp. 309-318

[11] $* * *$ - Directiva 2002/49/CE a Parlamentului European şi a Consiliului din 25 Iunie 2002 privind evaluarea şi gestiunea zgomotului ambiental

[12] *** - Sistem integrat de monitorizare acustică-video a poluării mediului cu zgomot, Contract de cercetare CEEX 132/2007 